\title{
Welfare and ethics
}

\section{Abuse: what drives abuse?}

\section{Paula Boyden}

Before exploring what drives abuse, one must be able to define what it means. When referring to the term abuse, although one might initially think of physical abuse, or so-called non-accidental injury (NAI), it is important to recognise that there are other forms of abuse. In order to consider a diagnosis of abuse, we must not only be able to recognise the abuse, but also to use the correct and appropriate terminology.

In the case of companion animals, the tried and tested child abuse terminology may be used to avoid confusion both within and between professions. The recognised categories of abuse are:

\section{- Physical \\ - Sexual \\ - Emotional \\ - Neglect}

It must be stressed that the diagnosis of NAl is a difficult challenge; both emotionally (we do not expect our patients to be intentionally hurt) and intellectually (it is a combination of factors that raises suspicion and that combination is variable). Furthermore, it may only be after a period of time that suspicions are aroused. Thanks to the work of Munro and Thrusfield we now have good diagnostic indicators for NAl in companion animals.

It should be no surprise that there are similarities in the abuse of humans and the abuse of animals - with the circumstances of the violence, the actions involved and the excuses offered. This is due to one common denominator: the human perpetrator

It may be hard to comprehend why anyone would deliberately hurt an animal and then seek veterinary attention. Whilst the primary responsibility of those in veterinary practice is to their animal patients, it is important to have an understanding behind the motivators for abuse, which includes the possibility of other abuse in the household. Indeed the perpetrator may use the family pet to exert power and control over their victim: 'If you don't do as I say, I will hurt the family pet...'

It must be borne in mind that whilst animal abuse may be an indicator of other abuse in the family, it is not a given. Equally, whilst the person presenting the pet may be a victim themselves, they might also be the perpetrator
We are not expected to cross our professional boundaries if other abuse, beyond that involving our animal patients, is involved. However, we ought to be prepared and able to show compassion if we suspect a client is also a victim of abuse, and to be able to signpost them to organisations where they might receive help and guidance.

Reporting cases of suspected abuse is not a mandatory requirement of veterinary surgeons or veterinary nurses, although every practice should have a protocol for dealing with such cases, including the provision of support for all members of the practice team

References available on request.

\section{KEY LEARNING OBJECTIVES}

- To understand the different types of abuse

- To be familiar with the diagnostic pointers for non-accidental injury (NAI) and consider it as a possible differential diagnosis

- To be aware of the link between violence to people and violence to animals

\section{MULTIPLE CHOICE QUESTIONS}

1. Which of the following is a synonym of non-accidental injury?
(A) Physical abuse
(B) Sexual abuse
(C) Emotional abuse
(D) Neglect

2. Which of the following is a diagnostic indicator for NAI?

(A) History consistent with the injury

(B) Repetitive injuries

(C) Severity of the injury

(D) Different family members giving the same history

3. Which of the following most closely defines 'the link'?

(A) A person who perpetrates abuse always abuses animals and humans

(B) A person who perpetrates abuse will abuse animals or humans, not both

(C) A person who perpetrates abuse in animals is more likely to abuse humans, and vice versa

(D) There is no association between abuse of animals and abuse of humans

\section{Anthropomorphism: a step too far?}

\section{Franck Meijboom}

A world without animals is hard to imagine. We are faced with animals in many ways and in many contexts. Often we value, love or admire animals because of their being different from humans, e.g., an animal's capacity to run, to dig or to navigate. However, in many cases our relationship with animals starts in the idea that the animals mirror some or even many of our human characteristics and capacities. In these cases we recognise ourselves and we can relate to animals in spite of the differences. This raises two questions. First, there is the question whether the impression is correct, that animals are similar to us. To answer that question we need in-depth 